\section{Bandwidth engineering of photonic crystal waveguide bends}

P.I. Borel, L.H. Frandsen, A. Harpøth, J.B. Leon, H. Liu, M. Kristensen, W. Bogaerts, P. Dumon, R. Baets,

V. Wiaux, J. Wouters and S. Beckx

An effective design principle has been applied to photonic crystal waveguide bends fabricated in silicon-on-insulator material using deep UV lithography resulting in a large increase in the low-loss bandwidth of the bends. Furthermore, it is experimentally demonstrated that the absolute bandwidth range can be adjusted in a post-fabrication thermal oxidation process.

Introduction: The planar photonic crystal waveguide ( $\mathrm{PhCW}$ ) has numerous potential applications because of its unique capability to control the propagation of light by utilising the photonic bandgap (PBG) effect $[1,2]$. This effect allows the interaction between light and the PhCW to take place on a minute scale $[3,4]$. Thereby, the overall size of optical components based on PhCW structures may be greatly minimised and, correspondingly, device packing density increased. Recent advances in deep UV lithography at $248 \mathrm{~nm}$ [5] have made mass fabrication of optical ultra-compact $\mathrm{PhCW}$ devices viable by employing existing fabrication methods commonly encountered in the semiconductor electronics industry.

Research has now reached a level where existing fabrication technologies allow manufacture of $\mathrm{PhCW}$ structures with low propagation losses [6-9]. Hence, there is presently worldwide focus on the design and fabrication of $\mathrm{PhCW}$ structures possessing adequate bandwidths PhCW structures with $20-40 \mathrm{~nm}$ useful optical bandwidths have previously been demonstrated [3, 10, 11]. Recently, Borel et al. [12] have demonstrated that a new inverse design method, topology optimisation, can be utilised to dramatically increase the bandwidth of a $\mathrm{PhCW}$ component. However, some of the holes in these components have special sizes and shapes that currently cannot be manufactured using deep UV lithography and, thus, cannot currently be mass fabricated. Except for these topology-optimised structures, no bandgap-based PhCW components have been demonstrated with satisfactory performance in a broad wavelength range.

In this Letter, we present an alternative design strategy, which also leads to large bandwidths of PhCW bends, and that is suitable for fabrication utilising deep UV lithography. Furthermore, we demonstrate a simple experimental procedure employing thermal oxidation on how to tune the absolute position of the operational bandwidth for an already fabricated batch of samples.

Waveguide fabrication: The photonic crystal structures have been patterned using $248 \mathrm{~nm}$ deep UV lithography and transferred into a silicon-on-insulator material, with a $220 \mathrm{~nm}$ thick silicon-layer separated from the substrate by a $1 \mu \mathrm{m}$ thick silica-layer, employing a standard anisotropic reactive ion etch. The fabrication procedure is described in detail in $[5,13]$. The structures are defined in a triangular lattice of air holes extending through the top silicon-layer, with a lattice constant $\Lambda=435 \mathrm{~nm}$ and regular hole diameter $d=0.53 \Lambda$. This configuration gives a relatively large PBG below the silica-line [14] and allows TE-like singlemode propagation in the PhCWs. A missing line of holes in the $\Gamma \mathrm{K}$ direction of the triangular lattice forms the waveguides.

Bend design and results: Owing to the PBG effect in a $\mathrm{PhCW}$ component, light may be routed around sharp corners such as the simple $60^{\circ}$ bends displayed in Fig. 1. Note that in each bend one hole has been displaced [14]. However, in singlemode operation, discontinuities in the straight $\mathrm{PhCW}$ introduce large reflections at the interface between different sections of the PhCW. Discontinuities may also excite higher-order modes that are not necessarily guided in the $\mathrm{PhCW}$. As a result, the simple bends shown in Fig. 1 only have a $\sim 40 \mathrm{~nm}$ bandwidth region with high transmission for TE-polarised light as can be seen from the spectrum in Fig. 2 (dashed curve). The measured transmission spectrum for the structure shown in Fig. 1 has been normalised to a straight $\mathrm{PhCW}$ of equivalent propagation length in order to extract the bend loss. The experimental characterisation setup has been described in detail in [6].

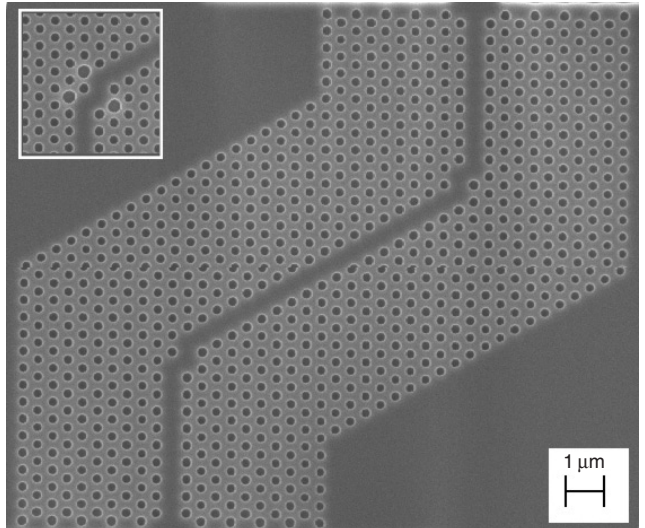

Fig. 1 Scanning electron micrograph of fabricated structure containing $60^{\circ}$ bends

One hole displaced at each bend

Inset: Scanning electron micrograph of how each $60^{\circ}$ bend modified

to improve bandwidth. Note that in this case one hole displaced and three holes enlarged at each bend

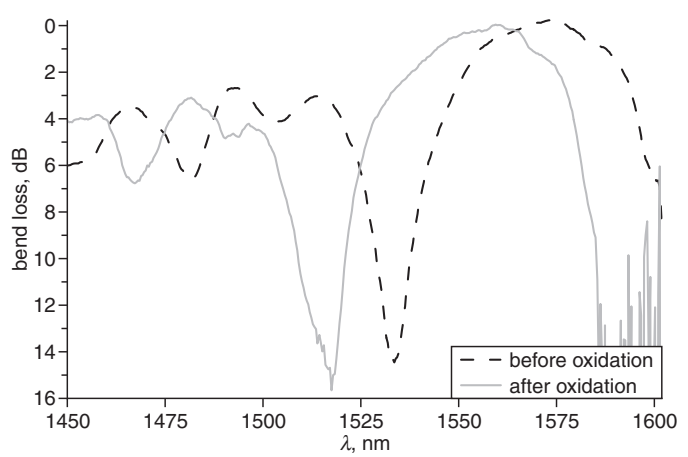

Fig. 2 Measured loss per bend for simple fabricated structure shown in Fig. 1 before and after thermal oxidation

To improve the bandwidth of the $\mathrm{PhCW} 60^{\circ}$ bends, the bend geometry was modified as shown in the inset of Fig. 1. Note that in addition to the displacement of one hole, the diameter of three holes has now been enlarged at each bend. The enlarged holes have a diameter $D=0.71 \Lambda$. This design is based on inspiration from microwave engineering [15]. The normalised transmission spectrum for TE-polarised light for a $60^{\circ}$ bend is obtained as before. The measured bend loss is displayed in Fig. 3 (dashed curve). Especially noteworthy is the $\sim 85 \mathrm{~nm}$ bandwidth with low bend loss around $\sim 1 \mathrm{~dB}$ and the square-like shape of the spectrum.

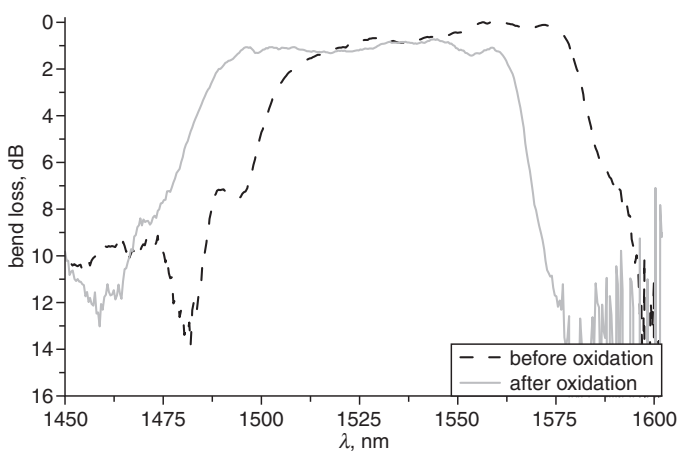

Fig. 3 Measured loss per bend for fabricated structure shown in inset of Fig. 1 before and after thermal oxidation

After the waveguide sample containing the above-mentioned $\mathrm{PhCW}$ structures had been fabricated and characterised, a thin layer $(\sim 10 \mathrm{~nm})$ of oxide was thermally grown on top of the sample. Thereby, the thickness of the core silicon layer and the hole diameters both changed slightly. These small oxidation changes introduce a blue shift of the spectra as seen in Figs. 2 and 3 (solid curves). In our experimental conditions it is observed that $\sim 1 \mathrm{~nm}$ of oxide blue shifts the spectrum about $1.3 \mathrm{~nm}$. It is seen that the spectral shift induced by the thermal 
oxidation essentially keeps the spectral features intact and merely changes the wavelength scale. $3 \mathrm{D}$ finite-difference time-domain calculations [14] have confirmed the observed frequency shifts caused by the thermal oxidation.

This thermal-oxidation procedure can conveniently be used in concert with vertical grating couplers [11]. When a batch of $\mathrm{PhCW}$ components have been fabricated, the spectral features can be assessed using the grating couplers to characterise the samples before the wafer is cleaved and the samples are packaged. If necessary the spectral features for the full batch can be shifted utilising thermal oxidation.

Conclusion: Planar $\mathrm{PhCW} 60^{\circ}$ bends have been fabricated in siliconon-insulator material utilising deep UV lithography. A simple design method has been utilised to increase the bandwidth by more than a factor of two. The manufactured $\mathrm{PhCW}$ bends have been designed with sufficient tolerances for fabrication with standard deep UV lithography. Furthermore, it has been demonstrated that thermal oxidation can be used in a post-processing step to precisely blue shift the spectral features a required amount.

Acknowledgments: This work was supported in part by the European IST project PICCO (photonic integrated circuits using photonic crystal optics) and the Danish Technical Research Council project PIPE (planar integrated PBG elements). W. Bogaerts and P. Dumon thank the Flemish Institute for the Industrial Advancement of Scientific and Technological Research (IWT) for a specialisation grant.

\section{(C) IEE 2004}

Electronics Letters online no: 20046206

doi: 10.1049/el:20046206

P.I. Borel, L.H. Frandsen, A. Harpøth, J.B. Leon, H. Liu and M. Kristensen (Research Center COM, Technical University of Denmark, Building 345V, DK-2800, Kgs. Lyngby, Denmark)

W. Bogaerts, P. Dumon and R. Baets (IMEC, Ghent University, Sint-Pietersnieuwstraat 41, 9000 Gent, Belgium)
V. Wiaux, J. Wouters and S. Beckx (IMEC vzw., Silicon Processing Technology Division, Kapeldreef 75, 3001 Leuven, Belgium)

\section{References}

1 Yablonovitch, E.: 'Inhibited spontaneous emission in solid-state physics and electronics', Phys. Rev. Lett., 1987, 58, pp. 2059-2062

2 John, S.: 'Strong localization of photons in certain disordered dielectric superlattices', Phys. Rev. Lett., 1987, 58, pp. 2486-2489

3 Thorhauge, M., Frandsen, L.H., and Borel, P.I.: 'Efficient photonic crystal directional couplers', Opt. Lett., 2003, 28, pp. 1525-1527

4 Song, B.-S., Noda, S., and Asano, T.: 'Photonic devices based on in-plane hetero photonic crystals', Science, 2003, p. 1537

5 Bogaerts, W., et al.: 'Fabrication of photonic crystals in silicon-oninsulator using 248-nm deep UV lithography', IEEE J. Sel. Top. Quantum Electron., 2002, 8, pp. 928-934

6 Borel, P.I., et al.: 'Efficient propagation of TM polarized light in photonic crystal components exhibiting band gaps for TE polarized light', Opt. Express, 2003, 11, pp. 1757-1762

7 McNab, S.J., Moll, N., and Vlasov, Y.A.: 'Ultra-low loss photonic integrated circuit with membrane-type photonic crystal waveguides', Opt. Express, 2003, 11, pp. 2927-2939

8 Sugimoto, Y., et al.: 'Low propagation loss of $0.76 \mathrm{~dB} / \mathrm{mm}$ in GaAsbased single-line-defect two-dimensional photonic crystal slab waveguides up to $1 \mathrm{~cm}$ in length', Opt. Express, 2004, 12, pp. 1090-1096

9 Notomi, M., et al.: 'Waveguides, resonators and their coupled elements in photonic crystal slabs', Opt. Express, 2004, 12, pp. 1551-1561

10 Frandsen, L.H., et al.: 'Ultra-low-loss 3-dB photonic crystal waveguide splitter', Opt. Lett., 2004, 29, (14), pp. 1623-1625

11 Taillaert, D., et al.: 'A compact two-dimensional grating coupler used as a polarization splitter', IEEE Photonics Technol. Lett., 2003, 15, pp. 1249-1251

12 Borel, P.I., et al.: 'Topology optimization and fabrication of photonic crystal structures', Opt. Express, 2004, 12, pp. 1996-2001

13 Bogaerts, W., et al.: 'Basic structures for photonic integrated circuits in silicon-on-insulator', Opt. Express, 2004, 12, pp. 1583-1591

14 Lavrinenko, A., et al.: 'Comprehensive FDTD modelling of photonic crystal waveguide components', Opt. Express, 2004, 12, pp. 234-248

15 Harvey, A.F.: 'Microwave engineering' (Academic Press, London, 1983) 